Ana I. Pereira - Florbela P. Fernandes .

João P. Coelho · João P. Teixeira .

Maria F. Pacheco · Paulo Alves ·

Rui P. Lopes (Eds.)

Optimization, Learning

Algorithms and Applications

First International Conference, OL2A 2021

Bragança, Portugal, July 19-21, 2021

Revised Selected Papers

望 Springer 


\section{Editors}

Ana I. Pereira

Instituto Politécnico de Bragança

Bragança, Portugal

João P. Coelho

Instituto Politécnico de Bragança

Bragança, Portugal

Maria F. Pacheco

Instituto Politécnico de Bragança

Bragança, Portugal

Rui P. Lopes (D)

Instituto Politécnico de Bragança

Bragança, Portugal
Florbela P. Fernandes (D)

Instituto Politécnico de Bragança

Bragança, Portugal

João P. Teixeira (1D)

Instituto Politécnico de Bragança

Bragança, Portugal

Paulo Alves

Instituto Politécnico de Bragança

Bragança, Portugal

ISSN 1865-0929

ISSN 1865-0937 (electronic)

Communications in Computer and Information Science

ISBN 978-3-030-91884-2

ISBN 978-3-030-91885-9 (eBook)

https://doi.org/10.1007/978-3-030-91885-9

\section{(C) Springer Nature Switzerland AG 2021}

This work is subject to copyright. All rights are reserved by the Publisher, whether the whole or part of the material is concerned, specifically the rights of translation, reprinting, reuse of illustrations, recitation, broadcasting, reproduction on microfilms or in any other physical way, and transmission or information storage and retrieval, electronic adaptation, computer software, or by similar or dissimilar methodology now known or hereafter developed.

The use of general descriptive names, registered names, trademarks, service marks, etc. in this publication does not imply, even in the absence of a specific statement, that such names are exempt from the relevant protective laws and regulations and therefore free for general use.

The publisher, the authors and the editors are safe to assume that the advice and information in this book are believed to be true and accurate at the date of publication. Neither the publisher nor the authors or the editors give a warranty, expressed or implied, with respect to the material contained herein or for any errors or omissions that may have been made. The publisher remains neutral with regard to jurisdictional claims in published maps and institutional affiliations.

This Springer imprint is published by the registered company Springer Nature Switzerland AG The registered company address is: Gewerbestrasse 11, 6330 Cham, Switzerland 


\section{Preface}

The volume CCIS 1488 contains the refereed proceedings of the International Conference on Optimization, Learning Algorithms and Applications (OL2A 2021), an event that, due to the COVID-19 pandemic, was held online.

OL2A 2021 provided a space for the research community on optimization and learning to get together and share the latest developments, trends, and techniques as well as develop new paths and collaborations. OL2A 2021 had more than 400 participants in an online environment throughout the three days of the conference (July 19-21, 2021), discussing topics associated to areas such as optimization and learning and state-of-the-art applications related to multi-objective optimization, optimization for machine learning, robotics, health informatics, data analysis, optimization and learning under uncertainty, and the Fourth Industrial Revolution.

Four special sessions were organized under the following topics: Trends in Engineering Education, Optimization in Control Systems Design, Data Visualization and Virtual Reality, and Measurements with the Internet of Things. The event had 52 accepted papers, among which 39 were full papers. All papers were carefully reviewed and selected from 134 submissions. All the reviews were carefully carried out by a Scientific Committee of $61 \mathrm{PhD}$ researchers from 18 countries.

July 2021

Ana I. Pereira 


\section{Organization}

\section{General Chair}

Ana Isabel Pereira

Polytechnic Institute of Bragança, Portugal

\section{Organizing Committee Chairs}

Florbela P. Fernandes

João Paulo Coelho

João Paulo Teixeira

M. Fátima Pacheco

Paulo Alves

Rui Pedro Lopes

\section{Scientific Committee}

Ana Maria A. C. Rocha

Ana Paula Teixeira

André Pinz Borges

Andrej Košir

Arnaldo Cândido Júnior

Bruno Bispo

Carmen Galé

B. Rajesh Kanna

C. Sweetlin Hemalatha

Damir Vrančić

Daiva Petkeviciute

Diamantino Silva Freitas

Esteban Clua

Eric Rogers

Felipe Nascimento Martins

Gaukhar Muratova

Gediminas Daukšys

Glaucia Maria Bressan

Humberto Rocha

José Boaventura-Cunha

José Lima

Joseane Pontes

Juani Lopéz Redondo
Polytechnic Institute of Bragança, Portugal

Polytechnic Institute of Bragança, Portugal

Polytechnic Institute of Bragança, Portugal

Polytechnic Institute of Bragança, Portugal

Polytechnic Institute of Bragança, Portugal

Polytechnic Institute of Bragança, Portugal 


Jorge Ribeiro
José Ramos
Kristina Sutiene
Lidia Sánchez
Lino Costa
Luís Coelho
Luca Spalazzi
Manuel Castejón Limas
Marc Jungers
Maria do Rosário de Pinho
Marco Aurélio Wehrmeister
Mikulas Huba

Michał Podpora

Miguel Ángel Prada

Nicolae Cleju

Paulo Lopes dos Santos

Paulo Moura Oliveira

Pavel Pakshin

Pedro Luiz de Paula Filho

Pedro Miguel Rodrigues

Pedro Morais

Pedro Pinto

Rudolf Rabenstein

Sani Rutz da Silva

Sara Paiva

Sofia Rodrigues

Sławomir Stępień

Teresa Paula Perdicoulis

Toma Roncevic

Vitor Duarte dos Santos

Wojciech Paszke

Wojciech Giernacki
Polytechnic Institute of Viana do Castelo, Portugal

NOVA University Lisbon, Portugal

Kaunas University of Technology, Lithuania

University of León, Spain

University of Minho, Portugal

Polytecnhic Institute of Porto, Portugal

Marche Polytechnic University, Italy

University of León, Spain

Université de Lorraine, France

University of Porto, Portugal

Federal University of Technology - Paraná, Brazil

Slovak University of Technology in Bratislava, Slovakia

Opole University of Technology, Poland

University of León, Spain

Technical University of Iasi, Romania

University of Porto, Portugal

University of Trás-os-Montes and Alto Douro, Portugal

Nizhny Novgorod State Technical University, Russia

Federal University of Technology - Paraná, Brazil

Catholic University of Portugal, Portugal

Polytechnic Institute of Cávado e Ave, Portugal

Polytechnic Institute of Viana do Castelo, Portugal

Friedrich-Alexander-University of Erlangen-Nürnberg, Germany

Federal University of Technology - Paraná, Brazil

Polytechnic Institute of Viana do Castelo, Portugal

Polytechnic Institute of Viana do Castelo, Portugal

Poznan University of Technology, Poland

University of Trás-os-Montes and Alto Douro, Portugal

University of Split, Croatia

NOVA University Lisbon, Portugal

University of Zielona Gora, Poland

Poznan University of Technology, Poland 
Optimizing Data Transmission in a Wireless Sensor Network Based on LoRaWAN Protocol

Thadeu Brito, Matheus Zorawski, João Mendes,

Beatriz Flamia Azevedo, Ana I. Pereira, José Lima, and Paulo Costa

Indoor Location Estimation Based on Diffused Beacon Network . . . . . . . . . 294 André Mendes and Miguel Diaz-Cacho

SMACovid-19 - Autonomous Monitoring System for Covid-19 . . . . . . . . 309 Rui Fernandes and José Barbosa

\section{Optimization in Control Systems Design}

Economic Burden of Personal Protective Strategies for Dengue Disease:

an Optimal Control Approach

Artur M. C. Brito da Cruz and Helena Sofia Rodrigues

ERP Business Speed - A Measuring Framework ................. 336 Zornitsa Yordanova

BELBIC Based Step-Down Controller Design Using PSO

João Paulo Coelho, Manuel Braz-César, and José Gonçalves

Robotic Welding Optimization Using A* Parallel Path Planning Tiago Couto, Pedro Costa, Pedro Malaca, Daniel Marques, and Pedro Tavares

\section{Deep Learning}

Leaf-Based Species Recognition Using Convolutional Neural Networks Willian Oliveira Pires, Ricardo Corso Fernandes Jr., Pedro Luiz de Paula Filho, Arnaldo Candido Junior, and João Paulo Teixeira

Deep Learning Recognition of a Large Number of Pollen Grain Types . . . . . 381 Fernando C. Monteiro, Cristina M. Pinto, and José Rufino

Predicting Canine Hip Dysplasia in X-Ray Images Using Deep Learning . . . . . . Daniel Adorno Gomes, Maria Sofia Alves-Pimenta, Mário Ginja, and Vitor Filipe

Convergence of the Reinforcement Learning Mechanism Applied to the Channel Detection Sequence Problem 


\title{
Leaf-Based Species Recognition Using Convolutional Neural Networks
}

\author{
Willian Oliveira Pires ${ }^{1}$, Ricardo Corso Fernandes Jr. ${ }^{1}$, \\ Pedro Luiz de Paula Filho ${ }^{1}$, Arnaldo Candido Junior ${ }^{1(凶)}$, \\ and João Paulo Teixeira ${ }^{2}$ (iD \\ 1 Federal University of Technology - Paraná, Medianeira Campus, Curitiba, Brazil \\ \{willianpires, ricjun\}@alunos.utfpr.edu.br, \{plpf, arnaldoc\}@utfpr.edu.br \\ ${ }^{2}$ Research Centre in Digitalization and Intelligent Robotics (CEDRI) - Instituto \\ Politecnico de Braganca, Braganca, Portugal \\ joaopt@ipb.pt \\ http://www.utfpr.edu.br/english, https://cedri.ipb.pt/
}

\begin{abstract}
Identifying plant species is an important activity in specie control and preservation. The identification process is carried out mainly by botanists, consisting of a comparison of already known specimens or using the aid of books, manuals or identification keys. Artificial Neural Networks have been shown to perform well in classification problems and are a suitable approach for species identification. This work uses Convolutional Neural Networks to classify tree species by leaf images. In total, 29 species were collected. This work analyzed two network models, Darknet-19 and GoogLeNet (Inception-v3), presenting a comparison between them. The Darknet and GoogLeNet models achieved recognition rates of $86.2 \%$ and $90.3 \%$, respectively.
\end{abstract}

Keywords: Deep learning $\cdot$ Leaf recognition $\cdot$ Tree classification

\section{Introduction}

Sustainability is an important concern in the context of business and governments in view of nature preservation. According to Shrivastava [12], both businesses and governments play an important role in nature's preservation. The identification process of forest species is important in this context, specially in the case of endangered species. Flora identification is currently made by botanists by comparing with already known species or with book guidance, manuals and identification keys. This comprises simple tasks as identifying whether the plant have flowers and fruits to more complex tasks, such as identifying the plant species by observing morphological attributes. For non-professionals, this process can be long and error prone, so an automated tool would save time and, possibly, plant species. The advancements made in computation, image processing techniques and pattern recognition unveiled new ways of specie identification. Deep learning based system are promising in this field, being helpful both for the professionals and non-professionals.

(C) Springer Nature Switzerland AG 2021

A. I. Pereira et al. (Eds.): OL2A 2021, CCIS 1488, pp. 367-380, 2021.

https://doi.org/10.1007/978-3-030-91885-9_27 
In this work, we used leaf images to train Convolutional Neural Netowrks $(\mathrm{CNNs})$ for the classification task. Two models were trained: GoogLeNet (Inception-v3) and Darknet-19, both implemented using Tensorflow. These models were chosen due to their light requirements when compared to other models, allowing for use in low-cost equipment during field research. The models can be used to identify species in natura.

This work is organized as follows. Section 2 presents an overview of CNNs, specie identification and related works. Section 3 presents the materials and methods used to train our models. Section 4 discusses the results. Finally, Sect. 5 contains the works conclusions.

\section{Background}

\subsection{Species Identification}

In order to realize plant species classification, botanists base themselves in vegetable taxonomy, analyzing characteristic group species by morphological similarities and genetic kinship links [10]. This process generated a field in botany called dendrology, which investigates woody plants identification, distribution and classification [5]. Dendrology scope includes root types, tree sizes, pilosity, shaft, as well as diagnostic elements (color, texture and structure). The shaft is a tree trunk part free of ramifications which can be of different types, shapes and bases.

Table 1 presents leaf components. The bases of leaf variety is its division type, as there are simple leaves, which presents a single leaf lamina (Fig. 1). There are also compound leaves, which present more than one leaflet, as shown in Fig. 2.

Table 1. Leaf Characteristics

\begin{tabular}{|c|c|}
\hline Scientific name & Description \\
\hline Leaf venation & Pattern of veins in the leaf \\
\hline Hairiness & Structures as hair in leaf surface \\
\hline Leaf arrangement & How leaves are arranged on a twig \\
\hline Stem & Plant structure that supports leaves, flowers and fruits \\
\hline Stipule & $\begin{array}{l}\text { A pair of small organs that may be attached to the twig on } \\
\text { either side of the petiole }\end{array}$ \\
\hline Leaf base & Part of leaf nearest to the petiole \\
\hline Leaf apexes & Part of leaf farthest from petiole \\
\hline Simple leaf & Leaf that has a single blade \\
\hline Compound leaf & Leaf that has two or more blades that are called leaflets \\
\hline Leaflets & Leaf subdivisions that are related to compound leaf \\
\hline Stalk & $\begin{array}{l}\text { A thin stem that supports a leaf and joints it to another part } \\
\text { of plant tree }\end{array}$ \\
\hline Rachis & Principal vein in the compound leaf, extension of stalk \\
\hline Bud & A small lump from which a flower, leaf or stem develops \\
\hline
\end{tabular}




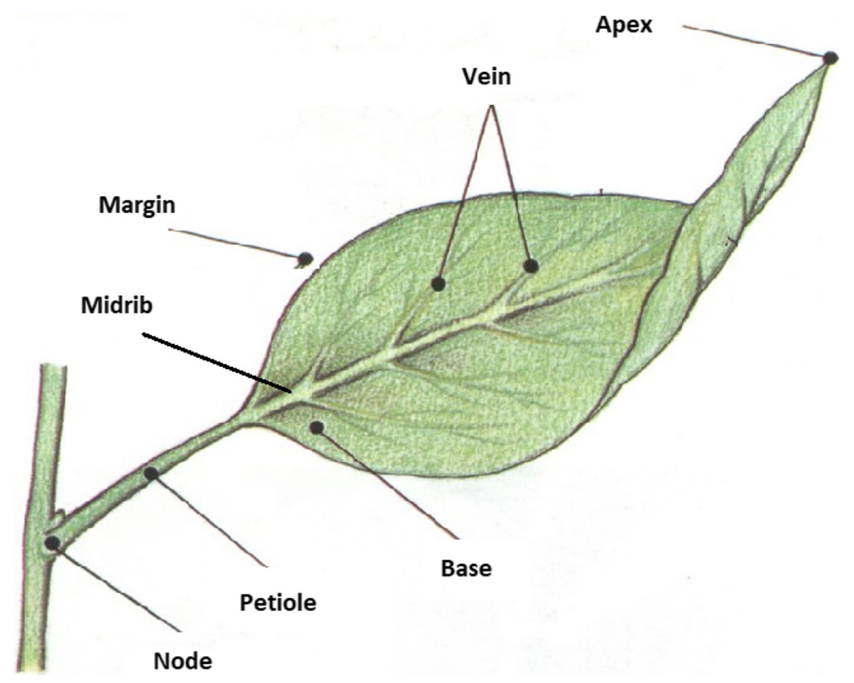

Fig. 1. Simple leaf [5]

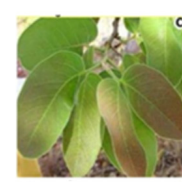

Bifoliate

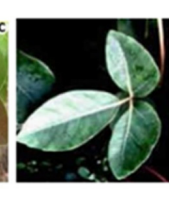

Trifoliate

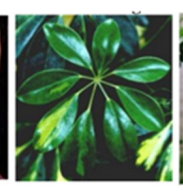

Palmate

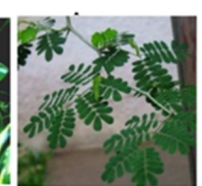

Bipinnate

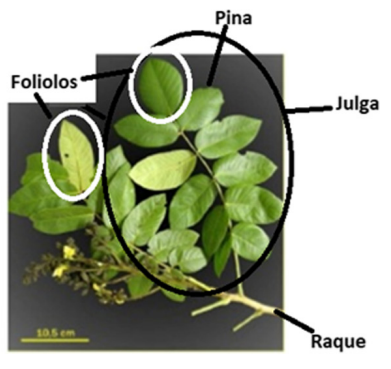

Unipinnate

Fig. 2. Compound leaf [5]

There are other important leaf elements, which generate a wide specie variety, such as leaf shape, tip (or apex), base and margin attributes, which can be used to differentiate plant species. Leafs also can be identified according to their phyllotaxis, which are divided in four types: alternate, spiral, opposite and whorled, shown in Fig. 3. This attribute is used before leaf extraction, as it shows how leaves are organized. Other attribute is leaf venation, which is divided in pinnate, reticulate, parallel, palmate and dichotomous (Fig.4).

All those characteristics are taken in account during species identification process and are the foundation of a widely used species identification method among botanists: dichotomous key, which is based in plant characteristics observation. Researchers compare characteristics of field extracted species with the characteristics of dichotomous keys, one by one, until matching with any of the registered species [10]. Table 2 presents a simple example using a dichotomous key to classify a leaf according to it's venation. 


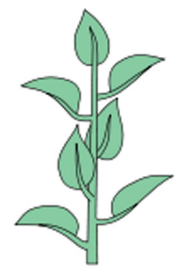

Alternate

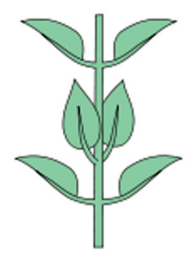

Spiral

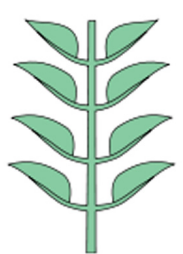

Opposite Whorled

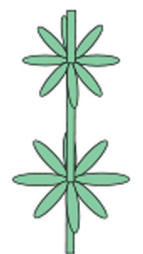

Fig. 3. Leaf phyllotaxis [5]

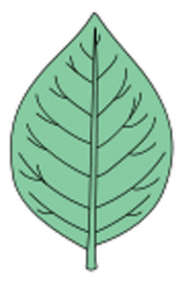

Pinnate

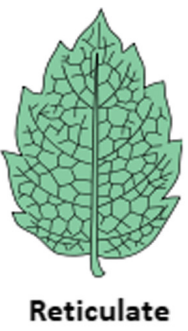

Reticulate

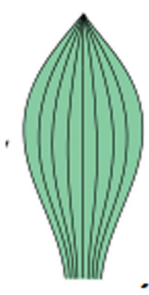

Parallel

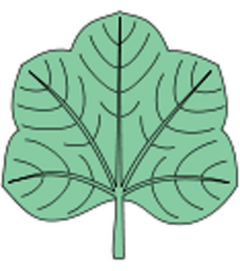

Palmate

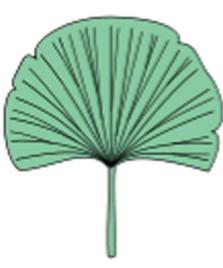

Dichotomous

Fig. 4. Leaf venation [5] onte

Table 2. Simple dichotomous key [5]

\begin{tabular}{|c|c|}
\hline $\begin{array}{l}\text { 1. Leaves with a single vein and not ramified } \\
\text { Leaves with more than a single venation }\end{array}$ & $\begin{array}{l}\text { Single main vein } \\
2\end{array}$ \\
\hline $\begin{array}{l}\text { 2. Leaves with more than a single vein and all } \\
\text { parallel between them }\end{array}$ & Parallel \\
\hline Leaves with non-parallel veins & 3 \\
\hline 3. Secondary veins originates from a main vein & Pinnate \\
\hline $\begin{array}{l}\text { Leaves with several main veins originating from } \\
\text { the petiole }\end{array}$ & Palmate \\
\hline
\end{tabular}

This is a fairly simple example, but in many cases characteristic selection might not be trivial and plant specie identification will usually involve more than a single characteristic. An example is Xanthosoma taioba, which is suitable for consumption and hard to classify, as it is very similar to Xanthosoma violaceum, which is not suitable for consumption. In Fig. 5, it becomes clear that differing two species is not always a simple task. 


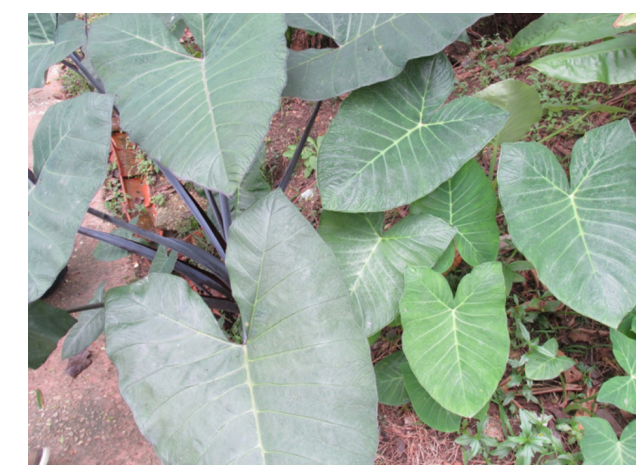

Fig. 5. The real Xanthosoma taioba (right), and Xanthosoma violaceum (left) [5]

\subsection{Convolutional Neural Networks}

In the context of machine learning, specifically in neural networks, Convolutional Neural Networks (CNNs) are a powerful model to analyze images. CNNs are popular in image processing since they are inspired by the visual cortex. These networks are based on the idea of specialized components inside a system with specific tasks, in a similar fashion as the visual cortex observed by [16]. This architecture is composed by a sequence of layers that tries to capture a hierarchy of increasingly sophisticated representations. Besides the input layer, which normally consists of an image with width, height and color depth (RGB red, green and blue channels), there are three typical layers: convolution layer, pooling layer and densely connected layer [15].

The first hidden layer in a CNN is usually a convolutional layer, which is composed by many feature maps (filters), capable of learning patterns as the training progresses [3]. Convolutional layers usually receive a two-dimensional or a collection of input two-dimensional and are widely used to process images. The layers are then submitted to a convolutional operation subject to parameters learned during the network training phase.

Each layer usually also performs a non-linear operation which greatly increases a model generalization capacity. This is done using an activation function. A popular function is the Rectified Linear Unit (ReLU), as it is a fast to calculate non-linear function. It replaces negative input values by zero [15]. An example as can be observed in (1), where $z$ represents the function input (a neuron input).

$$
\phi(z)= \begin{cases}0 & z \leq 0 \\ z & z>0\end{cases}
$$

After a convolution and activation function, it is common the use of a pooling layer. This technique aims to reduce the resulting matrix size, which diminishes the amount of neural network parameters to learn, contributing to avoid 
overfitting [15]. Max pooling is a pooling technique in which several inputs close to each other are replaced by a single value, the highest value in their neighbourhood.

Each consecutive layer are capable of representing more complex concepts than the previous layer. The last layers of a CNN usually are dense (or fully connected) layers, built on top of the convolutional layers. In case of CNNs for classification, the last layer outputs a $n$-dimensional vector, where $n$ is the total number of classes and each vector element is the predicted class probability for one of the available classes [3]. For classification, it is common the use of the Softmax function, which compares each output neuron response and return the results in the form of probability. This function is presented in (2), for the stimulus $z_{j}$ received by the $j$-nth output neuron.

$$
\phi\left(z_{j}\right)=\frac{e^{z_{j}}}{\sum_{k} e^{z_{k}}}
$$

These models are normally trained using the Backpropagation and Gradient Descent algorithms. To avoid overfitting, dropout technique can be used. Dropout approach consists on randomly removing neurons during training process [1].

\subsection{Darknet}

Darknet is a neural topology usually implemented in the YOLO framework. This framework allows real-time object detection and is able to identify objects in images and videos as presented in Table 3. In this work, we investigated Darknet19, an architecture composed of 19 convolutional layers interspersed with 5 more layers that apply max-pooling [3].

This network segments input image in $S x S$ frames, known as grids. To do this, it uses a divide and conquer strategy, making use of image segments to identify object position in addition to only identifying objects [2].

\subsection{GoogLeNet}

GoogLeNet is a CNN that became notorious after winning the 2014 Imagenet Competition. GoogLeNet engineer's objective was to enhance neural network computational efficiency while making it deeper and wider. The main feature in GoogLeNet is the inception module, which is based on the idea of using multiple convolutional filters [13] varying the kernel size used in the same convolutional layer. The enhanced inception module is presented in Fig. 6. 
Table 3. Darknet-19 [13]

\begin{tabular}{|c|c|c|c|}
\hline Type & Filters & Stride/Size & Output Dimension \\
\hline Convolutional & 32 & $3 \times 3$ & $224 \times 224$ \\
\hline Maxpool & & $2 \times 2 / 2$ & $112 \times 112$ \\
\hline Convolutional & 64 & $3 \times 3$ & $112 \times 112$ \\
\hline Maxpool & & $2 \times 2 / 2$ & $56 \times 56$ \\
\hline Convolutional & 128 & $3 \times 3$ & $56 \times 56$ \\
\hline Convolutional & 64 & $1 \times 1$ & $56 \times 56$ \\
\hline Convolutional & 128 & $3 \times 3$ & $56 \times 56$ \\
\hline Maxpool & & $2 \times 2 / 2$ & $28 \times 28$ \\
\hline Convolutional & 256 & $3 \times 3$ & $28 \times 28$ \\
\hline Convolutional & 128 & $1 \times 1$ & $28 \times 28$ \\
\hline Convolutional & 256 & $3 \times 3$ & $28 \times 28$ \\
\hline Maxpool & & $2 \times 2 / 2$ & $14 \times 14$ \\
\hline Convolutional & 512 & $3 \times 3$ & $14 \times 14$ \\
\hline Convolutional & 256 & $1 \times 1$ & $14 \times 14$ \\
\hline Convolutional & 512 & $3 \times 3$ & $14 \times 14$ \\
\hline Convolutional & 512 & $3 \times 3$ & $14 \times 14$ \\
\hline Convolutional & 256 & $1 \times 1$ & $14 \times 14$ \\
\hline Maxpool & & $2 \times 2 / 2$ & $7 \times 7$ \\
\hline Convolutional & 1024 & $3 \times 3$ & $7 \times 7$ \\
\hline Convolutional & 512 & $1 \times 1$ & $7 \times 7$ \\
\hline Convolutional & 1024 & $3 \times 3$ & $7 \times 7$ \\
\hline Convolutional & 512 & $1 \times 1$ & $7 \times 7$ \\
\hline Convolutional & 1024 & $3 \times 3$ & $7 \times 7$ \\
\hline $\begin{array}{l}\text { Convolutional } \\
\text { Avgpool } \\
\text { Softmax }\end{array}$ & 1000 & $\begin{array}{l}1 \times 1 \\
\text { Global }\end{array}$ & $\begin{array}{l}7 \times 7 \\
1000\end{array}$ \\
\hline
\end{tabular}

GoogLeNet is a 22 layer network, considering only convolutional layers, as shown in Table 4. The last layer uses the Softmax function to perform classification $[15]$.

\subsection{Related Work}

Many solutions based on deep learning have been used in specie identification problems, due to recent results using this technique. Other strategies can also be used, based on image processing and pattern recognition. These techniques use macro and microscopic characteristics of the image. For example, [8] classifies wood applying the following image characteristics extraction techniques: color 


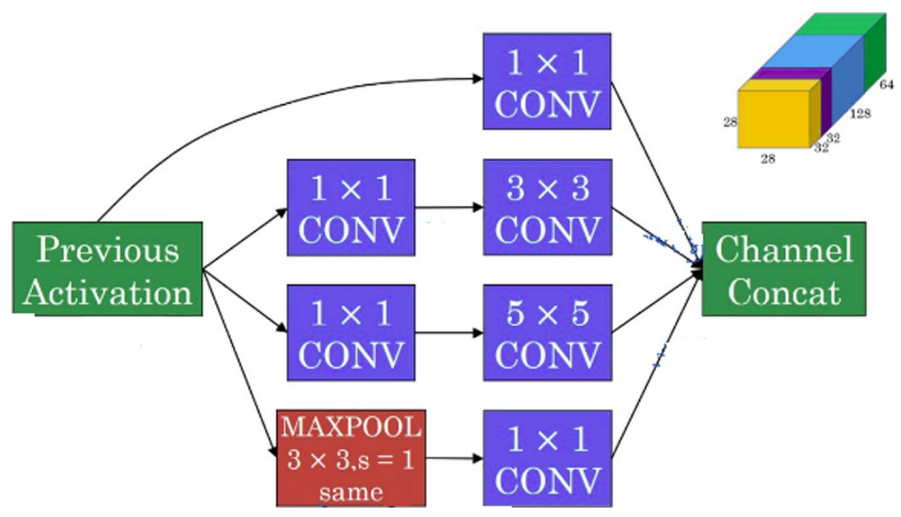

Fig. 6. Inception module [14]

Table 4. GoogLeNet's structure [13]

\begin{tabular}{l|l|l}
\hline Type & Filters/Stride & Output Dimension \\
\hline Convolution & $7 \times 7 / 2$ & $112 \times 112 \times 64$ \\
Max Pool & $3 \times 3 / 2$ & $56 \times 56 \times 64$ \\
Convolution & $3 \times 3 / 1$ & $56 \times 56 \times 192$ \\
Max Pool & $3 \times 3 / 2$ & $28 \times 28 \times 192$ \\
Inception (3a) & & $28 \times 28 \times 256$ \\
Inception (3b) & & $28 \times 28 \times 480$ \\
Max Pool & $3 \times 3 / 2$ & $14 \times 14 \times 480$ \\
Inception (4a) & & $14 \times 14 \times 512$ \\
Inception (4b) & & $14 \times 14 \times 512$ \\
Inception (4c) & & $14 \times 14 \times 512$ \\
Inception (4d) & & $14 \times 14 \times 512$ \\
Inception (4e) & & $14 \times 14 \times 832$ \\
Max Pool & $3 \times 3 / 2$ & $7 \times 7 \times 832$ \\
Inception (5a) & & $7 \times 7 \times 832$ \\
Inception (5b) & & $7 \times 7 \times 1024$ \\
Avg Pool & $7 \times 7 / 1$ & $1 \times 1 \times 1024$ \\
Dropout (40\%) & & $1 \times 1 \times 1024$ \\
Linear & & $1 \times 1 \times 1000$ \\
Softmax & & $1 \times 1 \times 1000$ \\
\hline & &
\end{tabular}

analysis, GLCM (Gray-Leval Co-occurence Matrix), border histogram, fractals, LBP (Local Binary Pattern), LPQ (Local Phase Quantization) and Gabor filter. This approach resulted in a recognition rate of $99.49 \%$ among 42 species.

Another work with related theme proposes analysis and identification of plant species based in texture characteristics extraction from microscopic leaf epidermis images [7]. Texture extraction techniques were used to analyze 32 species. This approach had 96\% success rate. By utilizing leaves, [11] applied image 
segmentation techniques for feature extraction. This was performed using the GLCM technique and feature vectors were extracted. The achieved recognition rate was around $75.4 \%$ by techniques like MLP (Multilayer Perceptron), SMO (Sequential Minimal Optimization) and LibSVM (Library for Support Vector Machines) as classifiers.

Using deep learning as main identification method, the strategy is basically to use CNNs to identify the best characteristic from the leaf to recognize a specie. From this strategy, [6] build CNNs being used for weeds control, which tries to detect a specie in the lawn. It was used $256 \times 256$ pixels images and the used architecture was AlexNet. The result was $75 \%$ precision [9].

There are other CNN approaches, [17] proposes the analysis of pictures taken from the top of farms, which demanded an extra detail preprocessing the image to enhance illumination before sending it to the network, that is composed of 5 convolutional layers with ReLU activation function and, at the end of the network, a Softmax function. Their experiment obtained $97.47 \%$ precision.

\section{Method}

\subsection{Data Collection}

Initially, leafs from 29 different species were collected. Species are listed in Sect. 4 (Table 4). For each species, 100 photos were taken on both sides. Images were obtained through the utilization of a photobooth proposed by [11]. It has 40 square centimeters and its internal contains led strips, which produces high luminosity with low energy costs. These leds can be feed using batteries or a $12 \mathrm{~V}$ power supply, and can be easily transported. To avoid reflexes on the images, internal walls were painted black, except for the bottom, which is white. The leaf is positioned on the bottom of the photobooth, where it is compressed by a glass pane, to keep it fixed and flat. This dataset is in the process of being public released.

After gathering enough photos, data augmentation techniques were used to artificially increase dataset size, as it is necessary to have many samples to train a Deep Neural Network. Data augmentation was done by using python 3.5.2 and Keras scripts to alter images, creating new ones. It was used Keras ImageDataGenerator class to generate new image samples from the original data, using the default values for the operations Rotation Range, Width Shift, Shear Range, Zoom Range, Horizontal and vertical Flip and Fill mode.

\subsection{Training}

The models were then trained with the augmented data and had their results evaluated, in order to improve performance. Both models, GoogLeNet and DarkNet19, were trained four times.

For GoogLeNet, we used the work of [13] as a reference to develop and train our network. Originally, the network was developed with batch size of 50, Reduce $1 \times 1$ of 104 and Dropout rate of 0.5 . 
Regarding Darknet-19, we used the python implementation Darkflow, which allow for the utilization of Darknet framework in Tensorflow [4]. Tiny-YOLO version 2 was used instead of YOLO version 3, as it was faster to train. As it was used 29 classes, the number of feature maps used in the last layer was 170, stride of size 1 and batch size of 64 , following the default configuration for Tiny-YOLO version 2.

\section{Tests and Results}

The original dataset were divided in 5,800 images for training and 580 for test. In order to improve results, both sets were subject to augmentation, generating 34,800 images for training and 2,900 for test. The augmented dataset contains independent training and test set, as no original image from training has an augmented version in test. Similarly, augmented images in training has no version in test.

Darknet model was first trained in the original dataset during 5.000 iterations, presenting True Positive $(\mathrm{TP})=414$, False Positive (FP) and False Negative $(\mathrm{FN})$ of 166 and harmonic mean of $71.3 \%$. After the first experiment, the model was than trained over the augmented dataset, during 18,000 iterations. Values presented by the network were $\mathrm{TP}=2502, \mathrm{FP}$ and $\mathrm{FN}$ of 398 and harmonic mean of $86.2 \%$. Precision and recall were calculated for each class. Results are presented on Table 4 and Fig. 7.

GoogLeNet experiments were analogous for Darknet-19. First the model was trained over the original dataset (5,800 images for training and 580 for test). This process was performed for 2,000 iterations. The network presented $\mathrm{TP}=461$, FP and FN of 119 and harmonic mean of $79.4 \%$. For the second experiment, (34,800 images for training and with 2,900 for validation) the iteration count was raised to 4,000. The network presented the following values: $\mathrm{TP}=2,633$, FP and FN of 267 and harmonic mean of 90.7\%. Values for precision, recall and harmonic mean were also calculated per class, presented on Table 4 and Fig. 8.

In the performed experiments, GoogLeNet had a better result than Darknet. The difference between both networks in precision, recall and harmonic mean were, respectively: 4.5, 4.8 and 4.7\%. GoogLeNet results are good, even with a broad and complicated dataset, as it were classified 29 classes, many of which are similar.

Analyzing results with and without data augmentation, it became clear the importance of a sufficient amount of data to work with CNNs. Differences between Darknet and GoogLeNet trained with small and sufficient amount of data was, respectively, $14.9 \%$ and $11.3 \%$. The developed data augmentation algorithm achieved it's objective, expanding the dataset without damaging samples. 
Table 5. Precision, recall and harmonic mean in details - YOLO and GoogLeNet

\begin{tabular}{|c|c|c|c|c|c|c|c|}
\hline & Scientific Name & $\begin{array}{l}\text { YOLO } \\
\text { Precision }\end{array}$ & $\begin{array}{l}\text { YOLO } \\
\text { Recall }\end{array}$ & $\begin{array}{l}\text { YOLO } \\
\text { F1 }\end{array}$ & $\begin{array}{l}\text { GoogLeNet } \\
\text { Precision }\end{array}$ & $\begin{array}{l}\text { GoogLeNet } \\
\text { Recall }\end{array}$ & $\begin{array}{l}\text { GoogLeNet } \\
\text { F1 }\end{array}$ \\
\hline 1 & Persea Americana & $63,0 \%$ & $64,2 \%$ & $72,6 \%$ & $73,0 \%$ & $100,0 \%$ & $84,3 \%$ \\
\hline 2 & Eriobotrya Japnica Lind & $57,0 \%$ & $100 \%$ & $72,6 \%$ & $75,0 \%$ & $100 \%$ & $85,7 \%$ \\
\hline 3 & Psidium Rufum & $100 \%$ & $80,0 \%$ & $88,8 \%$ & $100 \%$ & $74,0 \%$ & $85,1 \%$ \\
\hline 4 & Annona Montana & $96,0 \%$ & $56,1 \%$ & $70,8 \%$ & $98,0 \%$ & $64,0 \%$ & $77,4 \%$ \\
\hline 5 & Annona Squamosa & $97 \%$ & $100 \%$ & $98,4 \%$ & $100 \%$ & $100 \%$ & $100 \%$ \\
\hline 6 & Cojoba Arborea & $100 \%$ & $100 \%$ & $100 \%$ & $100 \%$ & $100 \%$ & $100 \%$ \\
\hline 7 & Coffea & $55,0 \%$ & $55,0 \%$ & $55,0 \%$ & $72,0 \%$ & $75,0 \%$ & $73,4 \%$ \\
\hline 8 & Pera Heteranthera & $95,0 \%$ & $100 \%$ & $97,4 \%$ & $98,0 \%$ & $82,3 \%$ & $89,4 \%$ \\
\hline 9 & Anacardium Occidentale & $100 \%$ & $100 \%$ & $100 \%$ & $100 \%$ & $100 \%$ & $100 \%$ \\
\hline 10 & Peltophorum dubium & $100 \%$ & $84,0 \%$ & $91,3 \%$ & $100 \%$ & $83,3 \%$ & $90,9 \%$ \\
\hline 11 & Nectandra Megapotamica & $98 \%$ & $100 \%$ & $98,9 \%$ & $94 \%$ & $100 \%$ & $96,9 \%$ \\
\hline 12 & Cerasus & $83,0 \%$ & $89,2 \%$ & $86,0 \%$ & $90,0 \%$ & $95,7 \%$ & $92,7 \%$ \\
\hline 13 & Prunus Serrulata & $78,0 \%$ & $100 \%$ & $87,6 \%$ & $91,0 \%$ & $100 \%$ & $95,2 \%$ \\
\hline 14 & Salix Babylonica & $100,0 \%$ & $100 \%$ & $100 \%$ & $82,0 \%$ & $100 \%$ & $90,1 \%$ \\
\hline 15 & Lle Paraguariensis & $81,0 \%$ & $100 \%$ & $89,5 \%$ & $80,0 \%$ & $100 \%$ & $88,8 \%$ \\
\hline 16 & Annona Coriácea & $94 \%$ & $80,3 \%$ & $86,6 \%$ & $100 \%$ & $94,3 \%$ & $97,0 \%$ \\
\hline 17 & Psidium Guajava & $83,0 \%$ & $76,8 \%$ & $79,8 \%$ & $94,0 \%$ & $88,6 \%$ & $91,2 \%$ \\
\hline 18 & Annona Muricata & $98 \%$ & $91,5 \%$ & $94,6 \%$ & $100 \%$ & $98,0 \%$ & $99,0 \%$ \\
\hline 19 & Syzygium Cumini & $84,0 \%$ & $85,7 \%$ & $84,8 \%$ & $97,0 \%$ & $100 \%$ & $98,4 \%$ \\
\hline 20 & Leucaena Leucocephala & $100 \%$ & $100 \%$ & $100 \%$ & $100 \%$ & $98 \%$ & $99 \%$ \\
\hline 21 & Citrus Limon & $72 \%$ & $76,5 \%$ & $74,2 \%$ & $76 \%$ & $100 \%$ & $86,3 \%$ \\
\hline 22 & Tibouchina Mutabilis & $100 \%$ & $83,3 \%$ & $90,9 \%$ & $100 \%$ & $90,0 \%$ & $94,7 \%$ \\
\hline 23 & Brunfelsia Uniflora & $81,0 \%$ & $81,0 \%$ & $81,0 \%$ & $90,0 \%$ & $100,0 \%$ & $94,7 \%$ \\
\hline 24 & Mangifera Indica & $97 \%$ & $100 \%$ & $98,4 \%$ & $98 \%$ & $79,6 \%$ & $87,8 \%$ \\
\hline 25 & Licania Tomentosa & $61,0 \%$ & $100 \%$ & $75,7 \%$ & $78,0 \%$ & $81,2 \%$ & $79,5 \%$ \\
\hline 26 & Dypsis Lutescens & $100 \%$ & $100 \%$ & $100 \%$ & $100 \%$ & $100 \%$ & $100 \%$ \\
\hline 27 & Paubrasilia Echinata & $86,0 \%$ & $100 \%$ & $92,4 \%$ & $89,0 \%$ & $100 \%$ & $94,1 \%$ \\
\hline 28 & Aspidosperma Polyneuron & $78,0 \%$ & $72,2 \%$ & $75,0 \%$ & $82,0 \%$ & $90,1 \%$ & $85,8 \%$ \\
\hline 29 & Eugenia uniflora & $65,0 \%$ & $100 \%$ & $88,8 \%$ & $76,0 \%$ & $77,5 \%$ & $78,7 \%$ \\
\hline
\end{tabular}




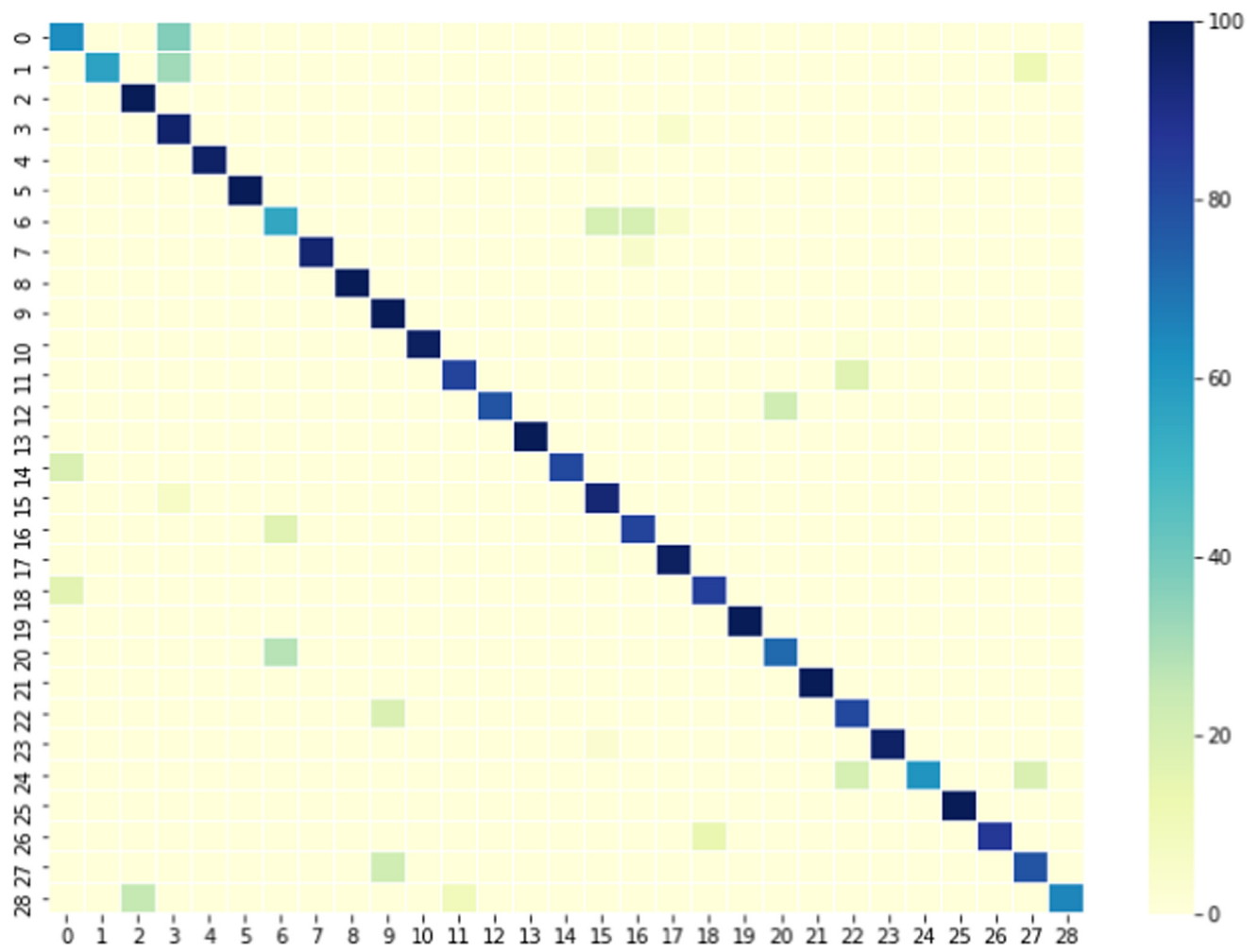

Fig. 7. Confusion Matrix Heatmap - Darknet19

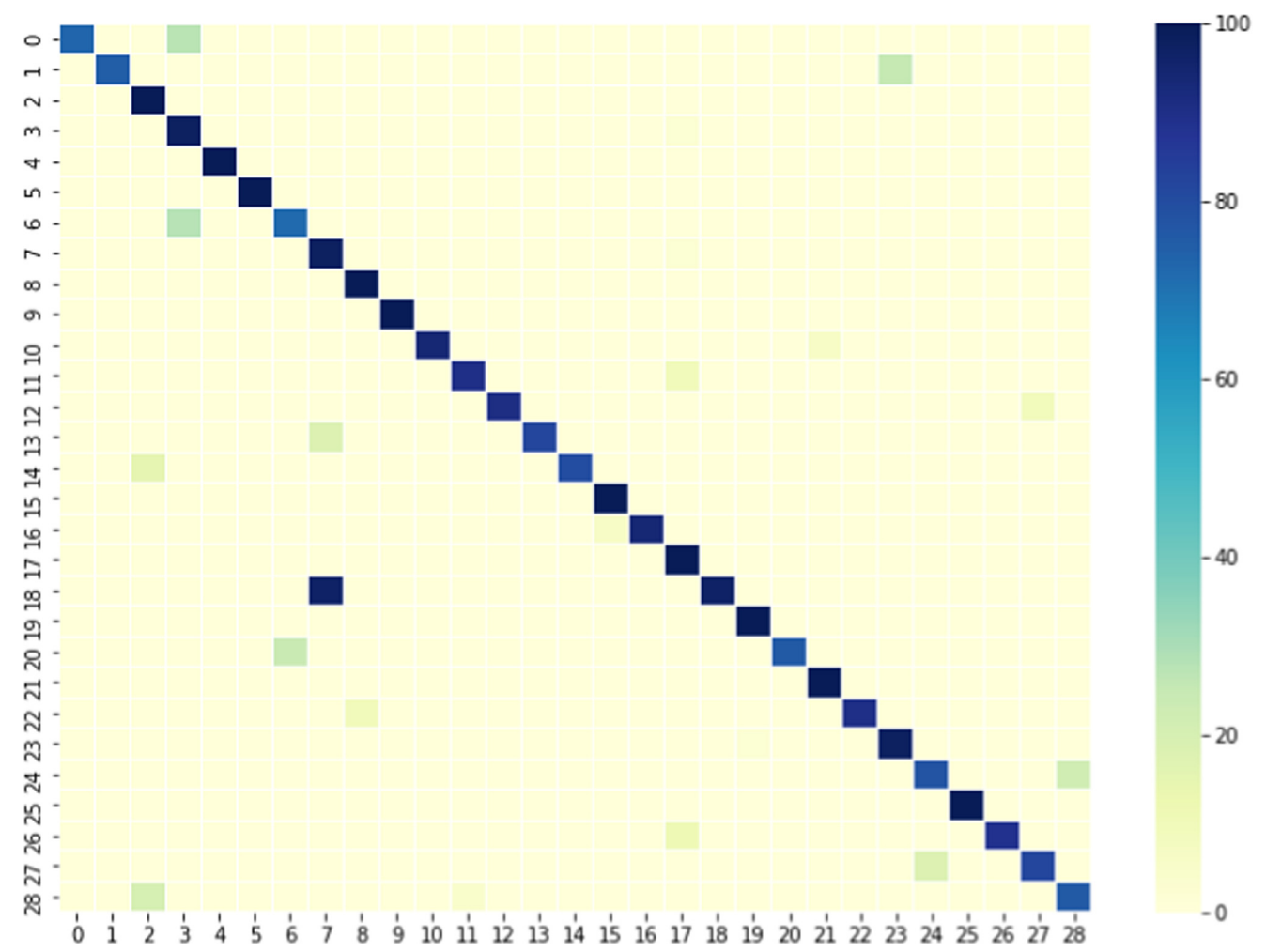

Fig. 8. Confusion Matrix Heatmap - GoogLeNet 


\section{Conclusions}

This work presented a comparison of Darknet-19 and GoogLeNet for tree species recognition using a dataset composed by leaf images from 29 different species, reaching recognition rates of $86.2 \%$ and $90.3 \%$, respectively. The obtained results demonstrates the viability of GoogLeNet and Darknet networks for classification. The models can be applied in field research, specially being used to identify species in natura.

For future works, we plan to test the models against images with leafs that were not removed from the tree. We also plan to use pre-trained Darknet networks in other platforms, as in smartphones aiming at practical uses of the model, comparing it with similar systems. YOLO have functionality for detecting objects in videos and android studio allows Tensorflow usage while associating a YOLOv2 training model. By using smartphone cameras, it is possible to develop an app to recognize plant species in video. Another suggestion would be to use the model in drones, as there is a huge amount of non registered plant species, in order to explore areas of limited access.

Acknowledgements. We gratefully acknowledge the support of NVIDIA Corporation with the donation of the GPU used in part of the experiments presented in this research.

\section{References}

1. Baldi, P., Sadowski, P.J.: Understanding dropout. In: 2013 Neural Information Processing Systems (2013). https://papers.nips.cc/paper/4878-understandingdropout

2. Farhadi, A., Girshick, R., Redmon, J.: You only look once: unified, real-time object detection. In: University of Washington. IEEE (2015)

3. Goodfellow, I., Bengio, Y., Courville, A., Bengio, Y.: Deep Learning, vol. 1. MIT press, Cambridge (2016)

4. Jones, R.P.: Darkflow (2018). https://medium.com/@richardpricejones/darkflow9bdc9f9b818e

5. Marchiori, J.N.C.: Elementos de Dendrologia. UFSM, Santa Maria (1995)

6. Mayo, S.J., Remagnino, P.: How deep learning extracts and learns leaf features for plant classification. Auton. Robot. 1-13 (2016)

7. Odemir, B., et al.: Leaf epidermis images for robust identification of plants. In: Instituto de Física de São Carlos, pp. 2-10 (2017)

8. de Paulo Filho, P.L.: Reconhecimento de espécies florestais através de imagens macroscópica. In: UFPR, pp. 9-48 (2012)

9. Pearlstein, L., Kim, M., Seto, M.: Convolutional neural network application to plant detection, based on synthetic imagery. In: Proceedings - Applied Imagery Pattern Recognition Workshop (2017). www.scopus.com

10. Pinheiro, A.L.: Fundamentos de taxonomia e dendrologia tropical. SIF, Santa Maria (2000)

11. Pires, W.O.: Reconhecimento de espécies florestais utilizando técnicas de processamento de imagem. In: 7ํㅗ Seminario de extenção e inovação, Londrina (2017) 
12. Shrivastava, P.: The role of corporations in achieving ecological sustainability. Acad. Manag. Rev. 20(4), 936-960 (1995)

13. Szegedy, C., Liu, W., Jia, Y.: Going deeper with convolutions. In: IEEE/ Boston, MA, USA. IEEE (2015)

14. Szegedy, C., Vanhoucke, V., Ioffe, S., Shlens, J., Wojna, Z.: Rethinking the inception architecture for computer vision. In: Proceedings of the IEEE Conference on Computer Vision and Pattern Recognition, pp. 2818-2826 (2016)

15. Vargas, A.C.G., Paes, A., Vasconcelos, N.: Um estudo sobre redes neurais convolucionais e sua aplicação em detecção de pedestres. In: IEEE/Conferencia Universidade Federal de Fluminence de Niterói. IEEE (2015)

16. Wurtz, R.H.: Recounting the impact of hubel and wiesel. Pattern Recogn. 32, 1-20 (2009). https://www.ncbi.nlm.nih.gov/pmc/articles/PMC2718241/

17. Yalcin, H., Razavi, S.: Plant classification using convolutional neural networks. In: 2016 5th International Conference on Agro-Geoinformatics, Agro-Geoinformatics 2016 (2016). www.scopus.com 